\title{
Rapid BAC selection for tol2-mediated transgenesis in zebrafish
}

\author{
Jeroen Bussmann ${ }^{1, *}$ and Stefan Schulte-Merker ${ }^{1,2, \dagger}$
}

\begin{abstract}
SUMMARY
The generation of zebrafish transgenic lines that express specific fluorophores in a cell- or tissue-specific manner is an important technique that takes full advantage of the optical clarity of the embryo. Identifying promoter fragments that faithfully recapitulate endogenous expression patterns and levels is often difficult and using large genomic DNA fragments, such as bacterial artificial chromosomes (BACs), makes the process of transgenesis less reliable. Here we provide a detailed protocol that allows for BAC selection and subsequent rapid modification through recombineering in Escherichia coli, resulting in BACs that can be injected into zebrafish embryos and, aided by tol2-mediated transgenesis, reliably yield stable transgenic lines. A number of BACs can be prepared in parallel, and injection of the BACs containing CFP/YFP/RFP or Gal4 cassettes allows for immediate testing of whether a particular BAC will yield the desired result. Furthermore, since injected embryos often show widespread expression, recombineered BACs provide an alternative to two-color in situ hybridizations: BACs injected into embryos of a different transgenic reporter line thus enable in vivo colocalization studies. Using this protocol, we have generated 66 stable lines for 23 different genes, with an average transgenesis rate above $10 \%$. Importantly, we provide evidence that BAC size shows no apparent correlation to the transgenesis rate achieved and that there are no severe position effects.
\end{abstract}

KEY WORDS: Imaging, Transgenesis, Zebrafish

\section{INTRODUCTION}

The transparency of the zebrafish embryo is one reason why this organism is among the mostly widely used experimental systems at present. Because of the small size of its embryos it is possible to visualize and analyze organs in their entirety, and also to follow single cell movements over time within an organ or the whole embryo. The paucity of antibodies in the zebrafish field, but more importantly the desire to perform in vivo imaging, have led to an ever increasing need to generate transgenic lines that allow an appreciation of the organ-specific, cell type-specific or genespecific expression of a fluorescent reporter (Koster and Fraser, 2001). In many cases this has been accomplished by generating transgenic lines that express GFP or RFP under the control of a specific promoter (or promoter fragment), but more recently the use of the Gal4/UAS binary system has become increasingly popular (Asakawa et al., 2008; Distel et al., 2009) as it allows, in combination with different UAS lines, an increased flexibility to control the expression of various reporter cassettes from the same promoter.

A persistent problem in generating transgenic lines has been the identification of functional promoter fragments, i.e. the identification of those elements in the $5^{\prime}$ region of a gene of interest that, when placed in front of a GFP cassette, will fully recapitulate the expression of the endogenous gene. Often, only certain aspects of the endogenous expression pattern are represented (Zhang and

\footnotetext{
${ }^{1}$ Hubrecht Institute - KNAW and Utrecht Medical Center, Uppsalalaan 8, 3584 CT, Utrecht, The Netherlands. 'Experimental Zoology Group, Department of Animal Sciences, Wageningen University, 6709 PG Wageningen, The Netherlands.

*Present address: Max Planck Institute for Molecular Biomedicine, Röntgenstraße 20, 48149, Münster, Germany

${ }^{\dagger}$ Author for correspondence (s.schulte@hubrecht.eu)
}

Accepted 22 June 2011
Rodaway, 2007), and frequently it is not possible at all to obtain the transgene of choice because of technical limitations in cloning sufficiently large promoter fragments that fully encompass all the necessary regulatory elements. One way around this particular problem is the use of bacterial artificial chromosomes (BACs) (Yang et al., 2006; Suster et al., 2009a), which can contain up to $350 \mathrm{~kb}$ of genomic DNA, although there are technical challenges in dealing with these rather large fragments and it is not straightforward to efficiently generate transgenic lines when using BACs. As a consequence, many laboratories have avoided using BACs, even though the advantages of the approach are obvious and have been amply demonstrated in other systems, such as the mouse (Giraldo and Montoliu, 2001; Van Keuren et al., 2009).

Here, we present a method that allows the generation of zebrafish BACs, which, through recombineering, can be modified to contain a fluorophore of choice at any given position in the BAC (most commonly the first translated ATG of a particular gene). The BAC is also modified to contain the long terminal repeats (LTRs) of the Medaka Tol2 transposon (Kawakami, 2007; Suster et al., 2009b; Suster et al., 2009a), which enable single-copy integration of the BAC into the zebrafish genome when co-injected with mRNA encoding the Tol 2 transposase into one-cell zygotes. The average transgenesis rate is higher than $10 \%$, which is a considerable improvement over conventional methods using BAC DNA. We have generated 66 lines with 23 different BACs using this protocol. Importantly, in many cases the analysis of injected embryos is immediately informative, as even transient expression from injected BACs is widespread and faithfully recapitulates endogenous gene expression. This is not only of immediate use to the researcher and in many cases allows circumvention of two-color in situ hybridization, but also allows an informed and fast decision to be made as to whether the newly generated BAC will be useful in generating the desired transgenic line. 


\section{MATERIALS AND METHODS}

A step-by-step protocol with a summary of required materials, a guide to BAC clone identification and a trouble-shooting guide are available at http://www.hubrecht.eu/research/schulte-merker/protocols.html and we strongly recommend that these data are consulted in combination with the information provided below.

\section{BAC recombineering overview}

Various methods have been developed - collectively called recombineering - in which laboratory strains of $E$. coli [which are normally deficient in homology-directed repair (HDR)] are designed to inducibly express genes encoding the HDR machinery. In this protocol, we employed a commercially available plasmid-based recombineering setup (GeneBridges, Germany) in which an arabinose-inducible promoter regulates the expression of an operon of HDR genes. This setup depends on a single plasmid (pRedET), which means that HDR can be performed in bacteria that already contain the $\mathrm{BAC}$, avoiding the need for BAC transformation into a specialized recombineering strain.

BAC DNA is stable using this system (see Fig. S1 in the supplementary material). Importantly, the efficiency of this recombineering setup is very high, which means small $(2 \mathrm{ml})$ bacterial culture volumes can be used, allowing for processing of up to eight different constructs in parallel. The pRedET plasmid contains a temperature-sensitive origin of replication (SC101), which replicates at $30^{\circ} \mathrm{C}$ but not at $37^{\circ} \mathrm{C}$. Therefore, after successful recombination, culturing at $37^{\circ} \mathrm{C}$ allows purification of $\mathrm{BAC}$ DNA without $\mathrm{pRedET}$ contamination.

The recombineering procedure we employed consists of three steps. First, BAC clones were transformed with the pRedET plasmid. Then, two Tol2 LTRs in opposing directions flanking an ampicillin resistance cassette (iTol2_amp) were inserted into the BAC vector backbone (Suster et al., 2009b). Finally, a reporter gene together with a kanamycin resistance cassette was inserted at the start ATG of the gene of interest. We have generated template plasmids encoding ten reporters: three different, bright and fastmaturing fluorophores [mCerulean (Rizzo et al., 2004)], Citrine (Griesbeck et al., 2001) and mCherry (Shaner et al., 2004), with cytoplasmic, nuclear or membrane localization. In addition, we cloned the transcriptional activator Gal4FF (Asakawa et al., 2008) for use with Gal4/UAS binary expression systems (see Fig. S2 in the supplementary material).

Isolated BAC DNA was co-injected with tol2 transposase mRNA to create first transient and, subsequently, stable transgenic zebrafish lines. Transient expression already gave a very reliable indication of the final expression of the stable transgenic reporter line. Therefore, within 12-14 days after the start of the procedure, an estimate could be made of the usefulness of the transgenic reporter for visualizing the organ, cell or protein of interest. A key step was the selection for raising of at least 20 embryos that show high and widespread expression of the injected reporter BAC.

This protocol has been optimized for the parallel preparation of multiple (up to eight) BAC constructs. In principle, the recombineering pipeline could be scaled up further, as, for example, described by Sarov et al. (Sarov et al., 2006). However, the hands-on time spent on embryo injection, founder identification and analysis will ultimately limit the number of transgenic lines that can be generated by a single person (we have had success with one person being responsible for preparing the BAC constructs, which were then handed over to the individual requester).

\section{BAC recombineering method}

\section{In silico BAC clone identification}

Clone identification was performed using Ensembl (http://www. ensembl.org) or the UCSC genome browser (http://genome.ucsc.edu). Various established BAC libraries exist that contain zebrafish genomic DNA of $35 \mathrm{~kb}$ to $250 \mathrm{~kb}$. The BAC end sequences of these libraries have been aligned to the zebrafish reference genome ('BAC end mapping') and these maps were used to identify clones that were likely to contain the gene(s) of interest. BAC clones were ordered from BACPAC resources (Oakland, CA, USA) or ImaGenes (Berlin, Germany). A step-by-step example of BAC clone identification can be found at http://www.hubrecht.eu/research/schulte-merker/protocols.html.

\section{Primer design}

Standard primers of $\sim 70$ nucleotides were used for targeting an iTol2_amp cassette to the $\mathrm{BAC}$ vector backbone; the choice of specific primers for this step depends on the sequence of the BAC vector (either pTarBAC derived or pIndigoBAC derived). Primer sequences for this step, including standard control primers to confirm correct recombination, are provided in Table S1 in the supplementary material.

Gene-specific primers were designed that contain 50 nucleotide homology arms around the start ATG of the gene of interest (positions -53 to -4 and +4 to +53 ) with $\sim 20$ nucleotide ends to amplify an XFP_kan/Gal4FF_kan cassette. Gene-specific primers and control primers were designed to amplify a 150-400 bp fragment upon correct recombination. Standard control primers within the XFP_kan/Gal4FF_kan cassettes are provided in Table $\mathrm{S} 1$ in the supplementary material.

A step-by-step example of gene-specific primer design can be found at http://www.hubrecht.eu/research/schulte-merker/protocols.html.

\section{Generation of targeting PCR products}

High-fidelity PCR reactions $(100 \mu \mathrm{l})$ were set up to generate targeting PCR products (templates for both targeting steps were generated at the same time): $1 \mu \mathrm{l} 5 \mathrm{ng} / \mu \mathrm{l}$ plasmid template, $20 \mu \mathrm{l} 5 \times$ Phusion HF Buffer (NEB), $2 \mu 110 \mathrm{mM}$ dNTPs, $2.5 \mu \mathrm{l}$ each of $10 \mu \mathrm{M}$ (pTarBAC/pIndigoBAC)_HA1_ iTol2 fw and (pTarBAC/pIndigoBAC) HA2 iTol2 rev recombineering primers (first targeting) or GeneX_HA1_(XFP or Gal4) fw and GeneX_HA2 kanR_rev recombineering primers (second targeting), $1 \mu 1$ Phusion DNA polymerase (NEB) and $71 \mu \mathrm{l}$ double-distilled (dd) $\mathrm{H}_{2} \mathrm{O}$. The following PCR conditions were used: initial denaturation at $98^{\circ} \mathrm{C}$ for 30 seconds; then 30 cycles of $98^{\circ} \mathrm{C}$ for 10 seconds, $58^{\circ} \mathrm{C}$ for 20 seconds, $72^{\circ} \mathrm{C}$ for 60 seconds; with a final extension at $72^{\circ} \mathrm{C}$ for 5 minutes.

Correct product sizes were confirmed on agarose gels. Then $2 \mu \mathrm{LpnI}$ (20 units) were added directly to the PCR reaction and incubated for 1 hour at $37^{\circ} \mathrm{C}$. PCR products were precipitated by adding $5 \mu 15 \mathrm{M} \mathrm{LiCl}$ and 300 $\mu 1100 \%$ ethanol, incubated at $-20^{\circ} \mathrm{C}$ for 30 minutes and subjected to centrifugation in a cooled bench-top centrifuge at maximal speed $(13,000$ $\mathrm{rpm}, 16,000 \mathrm{~g}$ ) for 30 minutes at $4^{\circ} \mathrm{C}$. The supernatant was discarded and the pellet washed with $70 \%$ ethanol. After 5 minutes centrifugation at maximal speed, the supernatant was discarded and the pellet dried at room temperature for 5-10 minutes. The pellets were resuspended in $21 \mu \mathrm{l}$ $\mathrm{ddH}_{2} \mathrm{O}$ and $1 \mu \mathrm{l}$ was used to measure the DNA concentration; $\mathrm{ddH}_{2} \mathrm{O}$ was then added to a final concentration of $500 \mathrm{ng} / \mu \mathrm{l}$.

\section{BAC confirmation and pRedET transformation}

Ordered BACs were streaked on an LB agar plate containing $11.3 \mu \mathrm{g} / \mathrm{ml}$ chloramphenicol $(\mathrm{Cm})$ and incubated overnight $(\mathrm{o} / \mathrm{n})$ at $37^{\circ} \mathrm{C}$ to obtain single colonies. One to three single colonies per BAC were picked and resuspended in $50 \mu \mathrm{l} \mathrm{ddH}_{2} \mathrm{O}$. Then $1 \mu \mathrm{l}$ was used for colony PCR to identify BACs containing the targeting sites and to test the control primers. Multiple PCRs were set up in 96-well PCR plates by combining, per well, $1 \mu 1$ suspended bacterial colony, $2 \mu 110 \times$ PCR buffer, $0.4 \mu 110 \mathrm{mM}$ dNTPs, $0.4 \mu \mathrm{l}$ each of $10 \mu \mathrm{M}$ GeneX_HA1_control_fw and GeneX_HA2_control_rev primers, $0.1 \mu \mathrm{l}$ Taq polymerase and $13.7 \mu \mathrm{l}$ $\mathrm{ddH}_{2} \mathrm{O}$. PCR was performed as: initial denaturation at $95^{\circ} \mathrm{C}$ for 5 minutes; 30 cycles of $94^{\circ} \mathrm{C}$ for 30 seconds, $58^{\circ} \mathrm{C}$ for 30 seconds, $72^{\circ} \mathrm{C}$ for 30 seconds; with a final extension at $72^{\circ} \mathrm{C}$ for 5 minutes. Orange $\mathrm{G}$ loading dye $(2.5 \mu \mathrm{l})$ was added to the PCR reactions and product sizes analyzed on a $2 \%$ agarose gel. If product sizes were as predicted, $20 \mu \mathrm{l}$ of the remaining bacterial colony suspension were added to $1 \mathrm{ml}$ of LB medium containing $\mathrm{Cm}$ and cultured $\mathrm{o} / \mathrm{n}$ at $37^{\circ} \mathrm{C}$.

A glycerol stock was generated by adding $500 \mu 150 \%$ glycerol to 500 $\mu \mathrm{l}$ of the $\mathrm{o} / \mathrm{n}$ culture for storage at $-80^{\circ} \mathrm{C}$. Forty microliters of the $\mathrm{o} / \mathrm{n}$ culture were transferred to $2 \mathrm{ml}$ of fresh LB medium containing $\mathrm{Cm}$ and cultured for 3 hours at $37^{\circ} \mathrm{C}$. Cultures were transferred to a $2 \mathrm{ml}$ microcentrifuge tube and centrifuged at $5000 \mathrm{rpm}(2300 \mathrm{~g})$ for 5 minutes. The supernatant was carefully removed and $1 \mathrm{ml}$ of ice-cold $\mathrm{dd}_{2} \mathrm{O}$ was added. Bacteria were resuspended by pipetting up and down twice. The bacteria were then recentrifuged at $5000 \mathrm{rpm}$ for 5 minutes and the pellet resuspended in $1 \mathrm{ml}$ of ice-cold $\mathrm{ddH}_{2} \mathrm{O}$. After a final centrifugation, the supernatant was mostly removed, leaving $\sim 50 \mu \mathrm{l}$ in the tube. Then, $1 \mu \mathrm{l}$ of 
$10 \mathrm{ng} / \mu \mathrm{l}$ pRedET plasmid was added and transferred to a prechilled 1-mm electroporation cuvette on ice (the transfer also served to mix and resuspend the bacterial pellet). Bacteria were electroporated using standard E. coli settings ( $1800 \mathrm{~V}, 25 \mu \mathrm{F}, 200 \Omega$, time constant of 4.8-5.2 mseconds), and, immediately after electroporation, $1 \mathrm{ml}$ of LB medium without antibiotics and containing $20 \mathrm{mM}$ D-glucose was added. Bacteria were transferred to a fresh culture tube and cultured at $37^{\circ} \mathrm{C}$ for 60 minutes. Then, $100 \mu$ of the cells were plated on LB plates containing $\mathrm{Cm}$ and 3.3 $\mu \mathrm{g} / \mathrm{ml}$ tetracycline (Tet) and incubated at $30^{\circ} \mathrm{C} \mathrm{o} / \mathrm{n}$. It could take up to 24 hours before colonies became visible $(>0.5 \mathrm{~mm}$ in diameter). A single colony was picked and transferred to fresh $\mathrm{LB}$ medium containing $\mathrm{Cm}$ and Tet and incubated at $30^{\circ} \mathrm{C}$ o/n (maximum 16 hours).

Insertion of iTol2_amp into the vector backbone

Five-hundred microliters of the $\mathrm{o} / \mathrm{n}$ culture were used to generate a glycerol stock (as above), and $150 \mu \mathrm{l}$ of the o/n culture were transferred to a culture tube containing $1.8 \mathrm{ml}$ of fresh LB medium with $\mathrm{Cm}$ and Tet and cultured for 2.5 hours at $30^{\circ} \mathrm{C}$. Sixty-seven microliters of $10 \%$ (w/v) L-(+)-arabinose were added to induce the expression of the HDR genes, and culturing was continued for 1 hour at $37^{\circ} \mathrm{C}$. The culture was transferred to a 2-ml centrifuge tube and processed for electroporation by washing twice in ice-cold $\mathrm{dd}_{2} \mathrm{O}$ in the same way as for the pRedET transformation. Before transferring to the electroporation cuvette, $1 \mu \mathrm{l}$ of $500 \mathrm{ng} / \mu \mathrm{l}$ iTol2_amp targeting PCR product was added. After electroporation, bacteria were incubated in LB medium without antibiotics but containing $20 \mathrm{mM} \mathrm{D}-(+)$-glucose for 45 minutes at $37^{\circ} \mathrm{C}$. Bacteria were plated on LB plates containing $\mathrm{Cm}$, Tet and $16.7 \mu \mathrm{g} / \mathrm{ml}$ ampicillin (Amp) and incubated at $30^{\circ} \mathrm{C} \mathrm{o} / \mathrm{n}$. It could take up to 24 hours before colonies were visible $(>0.5 \mathrm{~mm})$.

Correct iTol2 amp insertions were identified by colony PCR using the same protocol as described above for the BAC clone confirmation. Two to six colonies were picked per BAC ( $>95 \%$ had correct recombination). Primers used were pTarBAC/pIndigoBAC_HA1_control_fw and amp_HA1_control_rev for the left homology arm and pTarBAC/ pIndigoBAC_HA2_control_rev and amp_HA2_control_fw for the right homology arm. Product sizes were analyzed on a $2 \%$ agarose gel and only colonies in which both arms were correctly integrated were used. Products should be of the following sizes: pTarBAC_HA1_control_fw + amp_HA1_ control_rev, 405 bp; pTarBAC_HA2_control_rev + amp_HA2_control_fw, $317 \mathrm{bp}$; pIndigoBAC_HA1_control_fw + amp_HA1_control_rev, $420 \mathrm{bp}$; pIndigoBAC_HA2_control_rev + amp_HA2_control_fw, $32 \overline{7} \mathrm{bp}$.
Twenty microliters of the remaining bacterial colony suspension were added to $1 \mathrm{ml}$ of $\mathrm{LB}$ medium containing $\mathrm{Cm}$, Tet and Amp and cultured $\mathrm{o} / \mathrm{n}$ at $30^{\circ} \mathrm{C}$ (maximum 16 hours).

Insertion of XFP_kan or Gal4FF_kan at the start ATG of the gene of interest

Five-hundred microliters of the o/n culture were used to generate a glycerol stock (as above), and $150 \mu \mathrm{l}$ of the o/n culture were transferred to a culture tube containing $1.8 \mathrm{ml}$ of fresh LB medium with $\mathrm{Cm}$, Tet and Amp and cultured for 2.5 hours at $30^{\circ} \mathrm{C}$. Sixty-seven microliters of $10 \%(\mathrm{w} / \mathrm{v}) \mathrm{L}-(+)-$ arabinose was added to induce the expression of the HDR genes, and the culture was continued for 1 hour at $37^{\circ} \mathrm{C}$. The culture was transferred to a $2-\mathrm{ml}$ centrifuge tube and processed for electroporation by washing twice in ice-cold $\mathrm{ddH}_{2} \mathrm{O}$ in the same way as for the pRedET and iTol2 amp transformation. Before transfer to the electroporation cuvette, $1 \mu \mathrm{l}$ of 500 ng/ $\mu l$ XFP_kan/Gal4FF_kan targeting PCR product was added. After electroporation, bacteria were incubated in LB medium without antibiotics and containing $20 \mathrm{mM} \mathrm{D}-(+)$-glucose for 75 minutes at $37^{\circ} \mathrm{C}$. Bacteria were plated on LB plates containing Cm, Amp and $16.7 \mu \mathrm{g} / \mathrm{ml}$ kanamycin (Kan) and incubated at $37^{\circ} \mathrm{Co} / \mathrm{n}$.

Correct XFP_kan/Gal4FF_kan insertions were identified by colony PCR using the same protocol as described above for the BAC clone confirmation. Two to six colonies were picked per BAC $(>95 \%$ are expected to have correct recombination). Primers used were GeneX_HA1_control_fw and CFP/YFP/RFP/Gal4FF_HA1_control rev for the left homology arm and $\mathrm{CFP} / \mathrm{YFP} / \mathrm{RFP} / \mathrm{Gal} 4 \mathrm{FF}{ }^{-} \mathrm{HA}{ }_{2}^{-}$control fw and GeneX_HA2_control_rev for the right homology arm. Products sizes were analyzed on a $2 \%$ agarose gel and only colonies in which both arms were correctly integrated were used. Product size depended on the relative positions of the control primers to the homology arms. Twenty microliters of the remaining bacterial colony suspension were added to $1 \mathrm{ml}$ of LB medium containing $\mathrm{Cm}, \mathrm{Amp}$ and $\mathrm{Kan}$ and cultured $\mathrm{o} / \mathrm{n}$ at $37^{\circ} \mathrm{C}$ (maximum 16 hours)

\section{BAC DNA preparation}

Five-hundred microliters of the $\mathrm{o} / \mathrm{n}$ culture were used to generate a glycerol stock (as above) and the remaining culture was used to inoculate $100 \mathrm{ml}$ of LB medium containing Cm, Amp and Kan. The BAC DNA preparation was based on the HiPure Midiprep kit (Invitrogen), with modifications for BAC DNA isolation as described by the manufacturer. Precipitated DNA
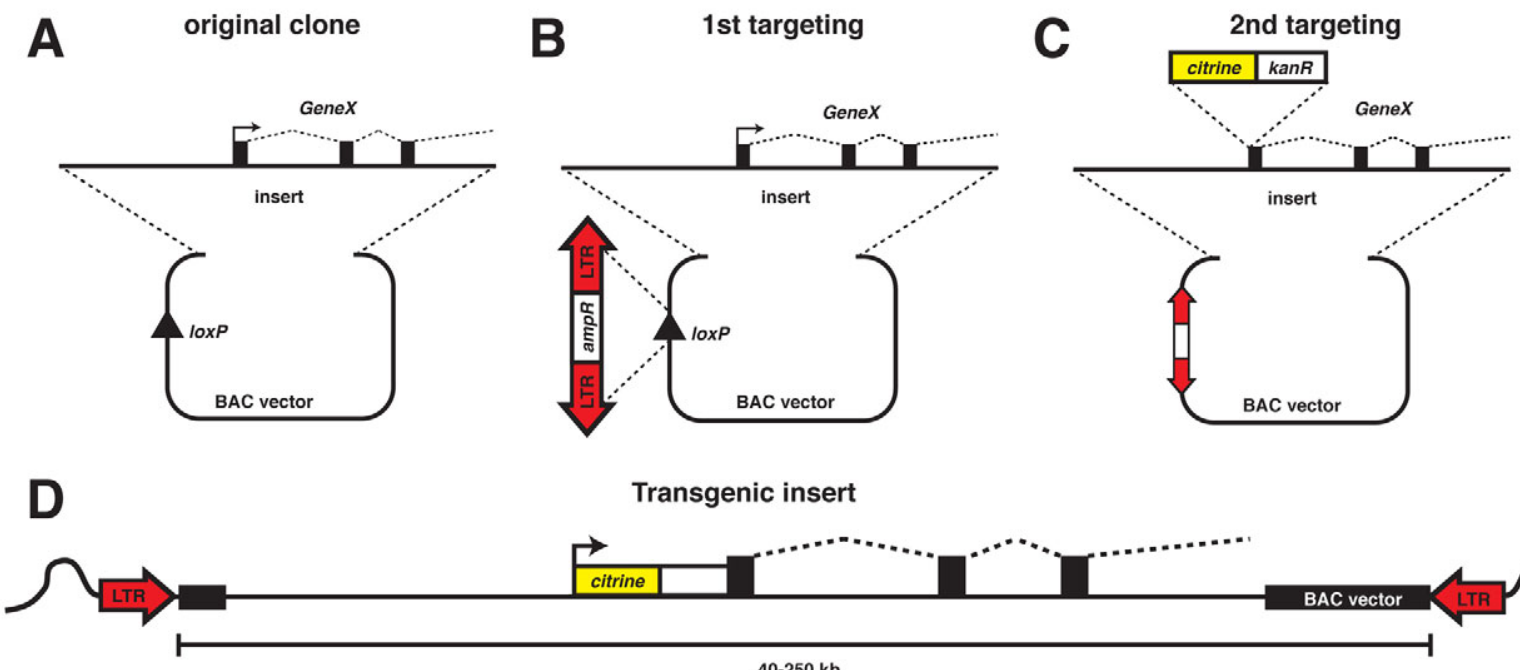

Transgenic insert

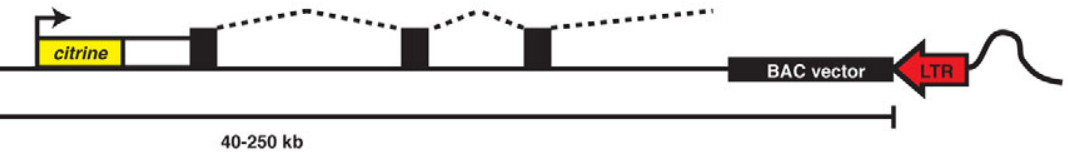

Fig. 1. Schematic representation of the BAC recombineering steps. (A,B) The unmodified BAC clone $(A)$ is first modified with a $P C R$ product containing inverted Tol2 LTRs (red arrows) and an ampicillin resistance gene (ampR), in the process deleting a loxP site from the vector backbone (B). (C) In the second recombineering step, a cassette containing a reporter gene (in this case citrine, yellow) and a kanamycin resistance gene (kanR) is inserted at the start codon of the gene of interest. (D) After to/2-mediated transgenesis, this results in the insertion of a 40-250 kb transgene into the zebrafish genome. The BAC vector sequences and Tol2 LTRs occupy the extremes of the transgenic inserts. The first and second targeting steps may be interchanged, but introducing the Tol2 LTRs first allows one to generate different reporter transgenes using the same BAC. 
Table 1. Transgenesis rates and copy number estimations for selected BAC transgenes

\begin{tabular}{|c|c|c|c|c|c|c|c|}
\hline Gene & BAC clone & Reporter & Adults screened & Founders identified & Copy number & Pattern & Insert (kb) \\
\hline$c x c / 12 b$ & CH73-291M8 & YFP & 26 & 2 & 1 & Yes & 97 \\
\hline entpd5 & CH73-213B8 & YFP & 19 & 4 & 1 & Yes & 82 \\
\hline cathepsin $K$ & CH73-114M20 & YFP & 13 & 2 & 2 & Yes & 78 \\
\hline cdh5 & CH73-357K2 & YFP & 8 & 3 & 1 & Yes & 163 \\
\hline cyp26b1 & DKEY-53014 & YFP & 1 & 1 & 1 & Yes & 171 \\
\hline arid1b & CH73-334P10 & YFP & 18 & 2 & 1 & Yes & 104 \\
\hline colll & CH73- 184B14 & RFP & 46 & 8 & $1-2$ & Yes/No* & 103 \\
\hline sox9b & $\mathrm{CH} 1073-175 \mathrm{H} 21$ & YFP & 9 & 1 & 1 & Yes & 35 \\
\hline prox1 & $\mathrm{CH} 211-174 \mathrm{~N} 9$ & YFP & 25 & 3 & 1 & Yes & 161 \\
\hline prox $1 b$ & CH73-247L15 & YFP & 10 & 3 & 1 & Yes & 116 \\
\hline Total & & & 175 & 29 & & & \\
\hline
\end{tabular}

Examples of ten different BAC transgenesis constructs. Adults screened refers to the number of adult putative founders screened by outcrossing. The copy number was estimated from the F1 transmission rate. Pattern refers to correlation of the reporter expression pattern with that of the endogenous gene (the asterisk indicates the one line that was an exception in this regard, see main text). Not all transgenic lines displayed in Fig. 2 are also listed here, where only those ten lines for which a complete set of parameters is known are shown.

was resuspended in $101 \mu \mathrm{l}$ and $1 \mu \mathrm{l}$ was used to measure the DNA concentration. $\mathrm{ddH}_{2} \mathrm{O}$ was added to a final concentration of $200 \mathrm{ng} / \mu \mathrm{l}$ and DNA was stored at $4^{\circ} \mathrm{C}$ for up to 2 weeks.

\section{Generation of transgenic lines}

Zebrafish maintenance and transgenesis were performed in accordance with the animal research guidelines of KNAW.

tol2 transposase mRNA was prepared by in vitro transcription from $X b a$ I-linearized $p D B 600$ (Balciunas et al., 2006) using the T3 mMessage mMachine kit (Ambion). RNA was purified using the RNeasy RNA purification kit (Qiagen), diluted to a final concentration of $100 \mathrm{ng} / \mu \mathrm{l}$ and divided into $5 \mu \mathrm{l}$ aliquots and stored at $-80^{\circ} \mathrm{C}$.

Fish were set up on the day before injections. For Gal4FF reporter constructs, we used $\operatorname{Tg}(U A S: G F P)^{n k u a s g f p l a}$. On the morning of the injections, a $5 \mu \mathrm{l}$ aliquot of BAC DNA $(200 \mathrm{ng} / \mu \mathrm{l})$ was prepared and a 5 $\mu \mathrm{l}$ aliquot of tol2 transposase mRNA $(100 \mathrm{ng} / \mu \mathrm{l})$ was thawed on ice. Zebrafish eggs were collected and, immediately prior to injection, BAC DNA and tol 2 transposase mRNA were mixed and loaded into an injection needle. The tip was broken and $2 \mathrm{nl}$ injected per embryo into the cytoplasm of 200 embryos at the 1- to 2-cell stage. A relatively large needle tip diameter and short injection pulses were used to limit shearing of the BAC DNA. Embryos were screened for malformations 8 and 24 hours after fertilization (hpf) and malformed or unfertilized embryos were discarded. Optimal transgenesis results were obtained when $30-50 \%$ of embryos were malformed at $24 \mathrm{hpf}$.

Embryos were screened for mosaic fluorescence in the expected tissues at $1-5$ days post-fertilization (dpf) and the $10 \%$ first-tier (brightest) $(n=10$ $20)$ and $40 \%$ second-tier ( $n=40-60)$ embryos were selected. These two batches were raised to adulthood.

Germline transmission was identified by mating sexually mature adult fish to wild-type fish and examining their progeny for fluorescence. Positive embryos were raised to adulthood and the line was maintained (and in some cases converted to a single-copy line) by outcrossing.

\section{In situ hybridization}

In situ hybridization was performed as previously described (SchulteMerker, 2002; Bussmann et al., 2007). RNA probes used were for $c d h 5$ (Larson et al., 2004) and cxcl12b (Miyasaka et al., 2007). The antisense $g f p / y f p$ probe was generated for 720 nucleotides of $y f p$ (citrine) coding sequence, which has greater than $99 \%$ nucleotide sequence identity to $g f p$ (egfp) and therefore could be used to detect both mRNAs.

\section{RESULTS}

We developed a protocol that is based on a recombination event that introduces an iTol 2 cassette into a BAC of choice. The BAC was then further modified (Fig. 1), as described in detail in the Materials and methods. The recombineering and transgenesis pipeline outlined in this protocol was tested and successfully used in the generation of 66 transgenic lines in two different laboratories, with transgenesis efficiencies ranging between $5 \%$ and $100 \%$ (founders identified out of total adult fish screened). Table 1 shows data for those 29 transgenic lines for which we have a complete dataset available, including copy numbers. To date, all BACs generated have been successfully converted into stable transgenic lines. Although it is mostly single-copy insertions that are recovered [in marked contrast to plasmid-based transgenesis regimes (Stuart et al., 1988; Winkler et al., 1991)], in some cases two independent copies were found in single F1 fish (Table 1), which required additional out-crossing to obtain fish with single-copy insertions. Transgenesis efficiency was found to be independent of BAC insert size (Fig. 2) in the size ranges of the currently available BAC libraries (up to $200 \mathrm{~kb}$ ). This effectively allows for greater flexibility in choosing which BAC can be used for generating a transgenic line. There was a strong correlation between known expression patterns and the expression of the respective reporters (Fig. 3A; see Fig. S3 in the supplementary material; see also below).

In contrast to smaller, promoter-based constructs, we found BAC transgenes to be highly resistant to position effects. Quantitative differences in fluorescence intensity between individual insertions of the same BAC were observed, however, potentially reflecting the chromatin status of the insertion sites. Only in one case among the 29 transgenic founders were qualitative differences in expression detected: here, the transgene showed only some of the expression domains that were expected based on prior knowledge of the endogenous mRNA expression pattern. However, seven

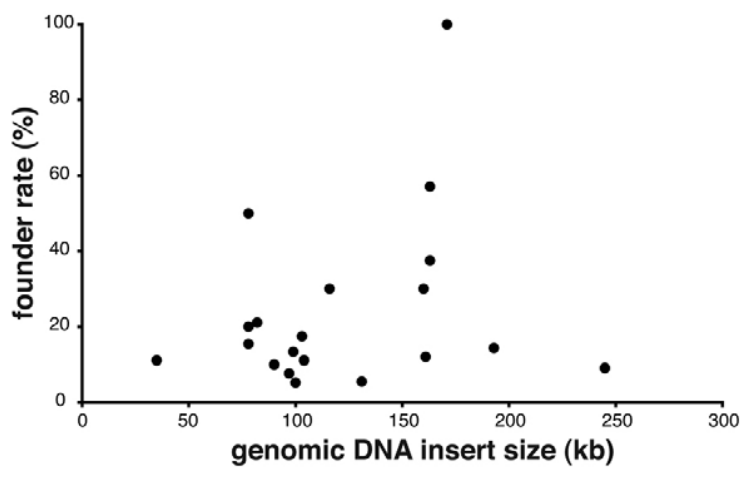

Fig. 2. BAC insert size does not affect transgenesis rate. Founder rates (percentage of germline founders among total adult fish screened) for constructs of different sizes (35-250 kb) indicate size independence of to/2-mediated BAC transgenesis within the typical size range of zebrafish BAC libraries. 

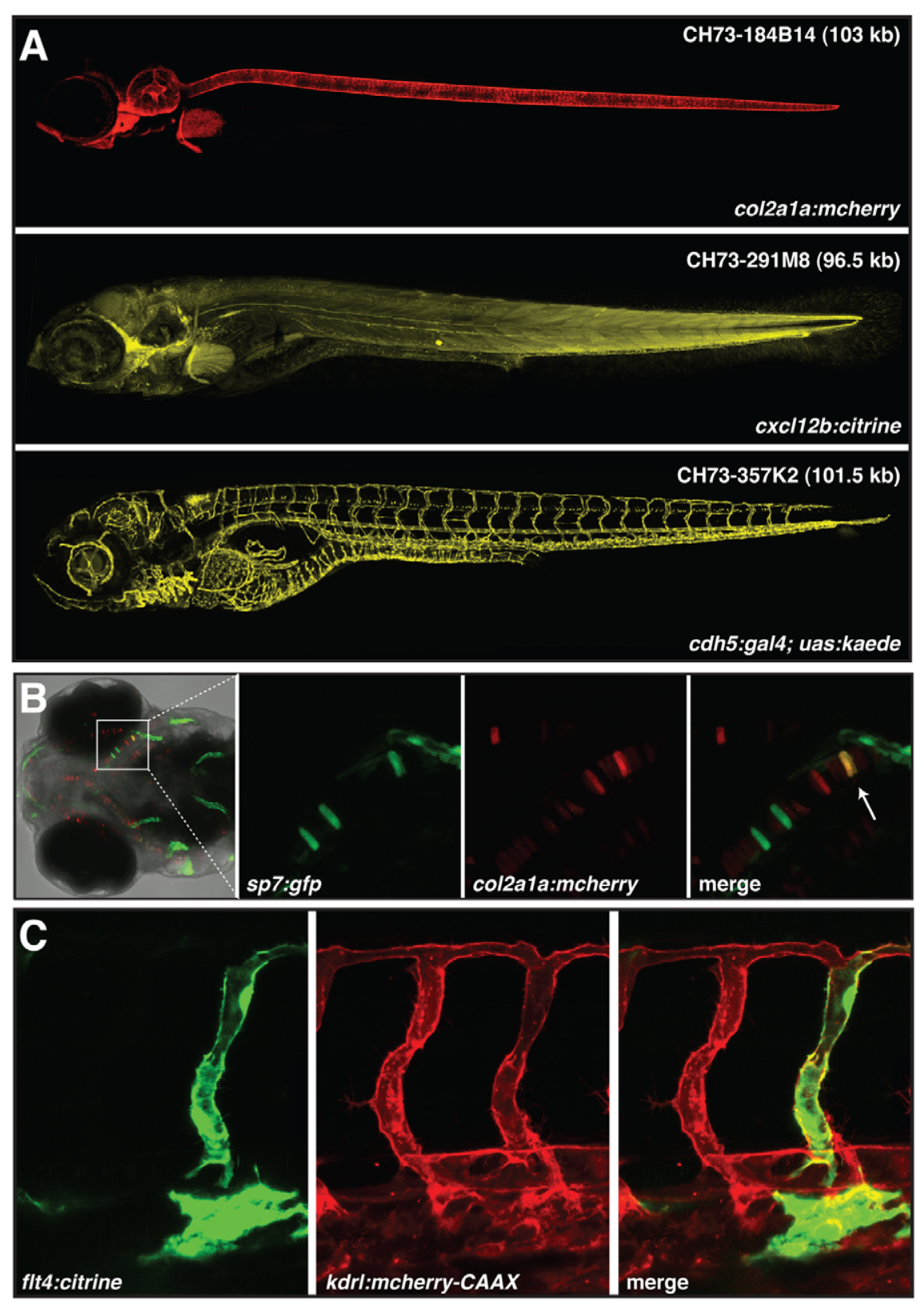

Fig. 3. Colocalization studies using recombineered BAC vectors. (A) Examples of stable, single-copy transgenic lines obtained using the described protocol. Lateral views of whole zebrafish embryos at 4-7 dpf. BAC clone IDs and predicted zebrafish genomic DNA insert sizes are indicated in the top right corner. (B) Dorsal view of the head of an sp7:gfp (Spoorendonk et al., 2008) transgenic zebrafish embryo at $5 \mathrm{dpf}$ injected with col2a1a:mCherry BAC DNA and to/2 transposase mRNA. Note the colocalization of GFP and mCherry in at least one cell (arrow). (C). Lateral view of the trunk of a kdrl:mCherry-CAAX (Hogan et al., 2009) transgenic embryo at $48 \mathrm{hpf}$ injected with a flt4:yfp (citrine) BAC and tol2 transposase mRNA. Individual YFP ${ }^{+}$endothelial cells (yellow) are detectable within the posterior caudal vein and intersegmental vessels. other lines using the same (col2ala) BAC showed identical patterns that were in full agreement with the in situ hybridization data. Since the transgenesis rate is high, we routinely aim to identify two or three independent founder lines in order to then isolate the strongest expression line.

\section{DISCUSSION}

In an attempt to establish a method that allows for scalable generation of recombineered BACs and transgenic lines, we have implemented a procedure comprising three distinct main phases: (1) in silico design of the experiment and selection of an appropriate BAC (see http://www.hubrecht.eu/research/schultemerker/protocols.html); (2) BAC recombineering (Fig. 1); and (3) the actual generation of the transgenic line. In using this method, we were particularly interested in addressing whether there is a negative correlation between BAC size and the rate of transgenesis, whether the recombineered BACs show sufficiently widespread expression to make them immediately useful for transient experiments (without the need to generate a transgenic line), and whether position effects are observable among different lines generated from the same BAC.

In previous experiments (Spoorendonk et al., 2008; Hogan et al., 2009), we have used I-SceI-mediated transgenesis (Grabher et al., 2004; Kimura et al., 2006) to perform BAC integration. In the limited number of BACs tested, we obtained maximal founder rates of $5 \%$, but also failed to identify founders in some cases. Therefore, the tol2 system appears to be more robust in terms of transgenesis efficiency. We presume that the use of the tol 2 system in conjunction with BACs results in early integration of BAC DNA into the fish genome, which in turn leads to a high number of embryos exhibiting an informative GFP or RFP expression pattern. This is of particular importance as it allows for immediate use of the recombineered BACs. As exemplified in Fig. 3B,C, the injection of a BAC into embryos that are transgenic for a different reporter, with a different color of fluorescence, enables colocalization studies in vivo with single-cell resolution. This is an important aspect because other 
available technologies used for colocalization studies are often technically challenging: the blue BCIP/NBT precipitate masks the red precipitate in those cases in which Fast Red is used, and if two different transcripts are to be detected in the same cell this poses a detection problem. Fluorescent in situ hybridization is an alternative here (Hauptmann and Gerster, 1994; Schulte-Merker, 2002; Bussmann et al., 2011), but this again poses technical problems. Furthermore, if late embryonic or larval stages are to be analyzed, and if the tissue in question is difficult for probes and antibodies to access (as is the case for cartilage and bone), in situ hybridizations are extremely difficult. In such cases, we have found BAC injection into an existing transgenic line to be a reliable alternative (Fig. 3B).

Although we have exclusively described the generation of zebrafish transcriptional reporter transgenic lines, with minor modifications many additional applications are possible. One example is the generation of C-terminal fusion proteins, which can be generated by changing the location of the homology arms from the $5^{\prime}$ - to the $3^{\prime}$-terminus of the coding sequence of the gene of interest. Another example is the generation of BAC transgenes in the final stages of a positional cloning project. Here, a tiling path of BAC transgenes (modified with the iTol2 amp cassette and a promoter-reporter transgene in the BAC vector backbone to select for transgenic founders) can be generated. If injected into eggs from the mutant strain in question, within one generation founders can be identified that are heterozygous for the causative mutation as well as being carriers of a specific transgene; these individuals may then be used for complementation crosses, eventually identifying the BAC that contains the wild-type copy of the mutated gene. We have used this approach twice and were successful in both cases (J.B. and S.S.-M., unpublished).

In summary, the method described here provides a scalable procedure for generating recombineered BACs that give high transgenesis rates when used in conjunction with tol 2 mRNA injection and independently of BAC size. Furthermore, we have observed position effects in only one case, and demonstrate here that BACs can be of immediate use for in vivo colocalization studies. Finally, this BAC transgenesis system could be extended to all vertebrate model systems in which tol2 transgenesis has been shown to function efficiently and for which well-annotated BAC libraries are available, such as Xenopus.

\section{Acknowledgements}

We thank Maximilian Suster and Koichi Kawakami for sharing unpublished information and reagents; the many members of the S.S.-M. group, past and present, for generating the transgenic lines that form the basis of this manuscript; L. Huitema, A. van Impel, I. Logister and B. Ponsioen for providing images for Fig. 3; and Josi Peterson-Maduro and Ive Logister for many improvements to the protocol. J.B. is grateful to Arndt Siekmann for support.

\section{Funding}

J.B. was funded by a Boehringer Ingelheim Fellowship and a stipend of the Vrienden van het Hubrecht Stichting during his PhD work. S.S.-M. is supported by KNAW.

\section{Competing interests statement}

The authors declare no competing financial interests.

\section{Author contributions}

J.B. designed the work and carried out and analyzed experiments. J.B. and S.S.-M. wrote the manuscript. S.S.-M. directed the work.

\section{Supplementary material}

Supplementary material for this article is available at

http://dev.biologists.org/lookup/suppl/doi:10.1242/dev.068080/-/DC1

References

Asakawa, K., Suster, M. L., Mizusawa, K., Nagayoshi, S., Kotani, T., Urasaki, A., Kishimoto, Y., Hibi, M. and Kawakami, K. (2008). Genetic dissection of neural circuits by Tol2 transposon-mediated Gal 4 gene and enhancer trapping in zebrafish. Proc. Natl. Acad. Sci. USA 105, 1255-1260.

Balciunas, D., Wangensteen, K. J., Wilber, A., Bell, J., Geurts, A., Sivasubbu, S., Wang, X., Hackett, P. B., Largaespada, D. A., Mclvor, R. S. et al. (2006). Harnessing a high cargo-capacity transposon for genetic applications in vertebrates. PLOS Genet. 2, e169.

Bussmann, J., Bakkers, J. and Schulte-Merker, S. (2007). Early endocardial morphogenesis requires Sc/Tal1. PLoS Genet. 3, e140.

Bussmann, J., Wolfe, S. A. and Siekmann, A. F. (2011). Arterial-venous network formation during brain vascularization involves hemodynamic regulation of chemokine signaling. Development 138, 1717-1726.

Distel, M., Wullimann, M. F. and Koster, R. W. (2009). Optimized Gal4 genetics for permanent gene expression mapping in zebrafish. Proc. Natl. Acad. Sci. USA 106, 13365-13370.

Giraldo, P. and Montoliu, L. (2001). Size matters: use of YACs, BACs and PACs in transgenic animals. Transgenic Res. 10, 83-103.

Grabher, C., Joly, J. S. and Wittbrodt, J. (2004). Highly efficient zebrafish transgenesis mediated by the meganuclease I-Scel. Methods Cell Biol. 77, 381 . 401.

Griesbeck, O., Baird, G. S., Campbell, R. E., Zacharias, D. A. and Tsien, R. Y. (2001). Reducing the environmental sensitivity of yellow fluorescent protein. Mechanism and applications. J. Biol. Chem. 276, 29188-29194.

Hauptmann, G. and Gerster, T. (1994). Two-color whole-mount in situ hybridization to vertebrate and Drosophila embryos. Trends Genet. 10, 266.

Hogan, B. M., Bos, F. L., Bussmann, J., Witte, M., Chi, N. C., Duckers, H. J. and Schulte-Merker, S. (2009). Ccbe1 is required for embryonic lymphangiogenesis and venous sprouting. Nat. Genet. 41, 396-398.

Kawakami, K. (2007). Tol2: a versatile gene transfer vector in vertebrates. Genome Biol. 8 Suppl. 1, S7.

Kimura, Y., Okamura, Y. and Higashijima, S. (2006). alx, a zebrafish homolog of $\mathrm{Chx} 10$, marks ipsilateral descending excitatory interneurons that participate in the regulation of spinal locomotor circuits. J. Neurosci. 26, 5684-5697.

Koster, R. W. and Fraser, S. E. (2001). Tracing transgene expression in living zebrafish embryos. Dev. Biol. 233, 329-346.

Larson, J. D., Wadman, S. A., Chen, E., Kerley, L., Clark, K. J., Eide, M. Lippert, S., Nasevicius, A., Ekker, S. C., Hackett, P. B. et al. (2004). Expression of VE-cadherin in zebrafish embryos: a new tool to evaluate vascular development. Dev. Dyn. 231, 204-213.

Miyasaka, N., Knaut, H. and Yoshihara, Y. (2007). Cxcl12/Cxcr4 chemokine signaling is required for placode assembly and sensory axon pathfinding in the zebrafish olfactory system. Development 134, 2459-2468.

Rizzo, M. A., Springer, G. H., Granada, B. and Piston, D. W. (2004). An improved cyan fluorescent protein variant useful for FRET. Nat. Biotechnol. 22, 445-449.

Sarov, M., Schneider, S., Pozniakovski, A., Roguev, A., Ernst, S., Zhang, Y., Hyman, A. A. and Stewart, A. F. (2006). A recombineering pipeline for functional genomics applied to Caenorhabditis elegans. Nat. Methods 3, 839844

Schulte-Merker, S. (2002). Looking at embryos. In Zebrafish: a Practical Approach (ed. C. Nüsslein-Volhard and R. Dahm), pp. 39-58. Oxford, UK: Oxford University Press.

Shaner, N. C., Campbell, R. E., Steinbach, P. A., Giepmans, B. N., Palmer, A. E. and Tsien, R. Y. (2004). Improved monomeric red, orange and yellow fluorescent proteins derived from Discosoma sp. red fluorescent protein. Nat. Biotechnol. 22, 1567-1572.

Spoorendonk, K. M., Peterson-Maduro, J., Renn, J., Trowe, T., Kranenbarg, S., Winkler, C. and Schulte-Merker, S. (2008). Retinoic acid and Cyp26b1 are critical regulators of osteogenesis in the axial skeleton. Development 135, 37653774.

Stuart, G. W., McMurray, J. V. and Westerfield, M. (1988). Replication, integration and stable germ-line transmission of foreign sequences injected into early zebrafish embryos. Development 103, 403-412.

Suster, M. L., Sumiyama, K. and Kawakami, K. (2009a). Transposon-mediated BAC transgenesis in zebrafish and mice. BMC Genomics 10, 477.

Suster, M. L., Kikuta, H., Urasaki, A., Asakawa, K. and Kawakami, K. (2009b). Transgenesis in zebrafish with the tol2 transposon system. Methods Mol. Biol. 561, 41-63.

Van Keuren, M. L., Gavrilina, G. B., Filipiak, W. E., Zeidler, M. G. and Saunders, T. L. (2009). Generating transgenic mice from bacterial artificial chromosomes: transgenesis efficiency, integration and expression outcomes. Transgenic Res. 18, 769-785

Winkler, C., Vielkind, J. R. and Schartl, M. (1991). Transient expression of foreign DNA during embryonic and larval development of the medaka fish (Oryzias latipes). Mol. Gen. Genet. 226, 129-140.

Yang, Z., Jiang, H., Chaichanasakul, T., Gong, S., Yang, X. W., Heintz, N. and Lin, S. (2006). Modified bacterial artificial chromosomes for zebrafish transgenesis. Methods 39, 183-188.

Zhang, X. Y. and Rodaway, A. R. (2007). SCL-GFP transgenic zebrafish: in vivo imaging of blood and endothelial development and identification of the initial site of definitive hematopoiesis. Dev. Biol. 307, 179-194. 